\title{
18. X-RAY MINERALOGY DATA, WESTERN INDIAN OCEAN-LEG 24 DEEP SEA DRILLING PROJECT ${ }^{1}$
}

\author{
J. C. Matti, I. Zemmels, and H. E. Cook, University of California, Riverside, California
}

\section{METHODS}

Semiquantitative determinations of the mineral composition of bulk samples, $2 \cdot 20 \mu$, and $<2 \mu$ fractions were performed according to the methods described in the reports of Legs 1 and 2 and in Appendix III of Volume IV. The mineral analyses of the $2-20 \mu$ and $<2 \mu$ fractions were performed on $\mathrm{CaCO}_{3}$-free residues.

The X-ray mineralogy results of this study are summarized in Tables 1 through 7 . The mineralogy data are presented in Tables 8 through 15. Sediment ages, lithologic units, and nomenclature of the sediment types in Tables 1 through 7 are from the DSDP Leg 24 hole summaries and from a subsequent update supplied by Dr. Peter Supko, DSDP. The stratigraphic position of samples submitted for X-ray diffraction analyses from Leg 24 are listed in Tables 1 through 7 . The sample depth (in meters) below the sea floor in Tables 1 through 7 identifies the samples as they are reported in Tables 8 through 15 . No samples were submitted for X-ray diffraction analysis from Site 237.

Several unidentified minerals were detected in Leg 24 samples. Their abundances were determined on a semiqualitative basis using a hypothetical mineral concentration factor of 3.0. Unidentified minerals are reported on a ranked, semiqualitative scale as outlined below:

Trace: $(<5 \%)$; diffraction pattern was weak and identification was made on the basis of two major diagnostic peaks.

Present: (5\%-25\%); a number of peaks of the mineral are visible in the diffraction pattern.

Abundant: $(25 \%-65 \%)$; diffraction peaks of the mineral are prominent in the total diffraction pattern, but the peaks of other minerals are of an equivalent intensity.

Major: (>65\%); the diffraction peaks of the mineral dominate the diffraction pattern.

Although a certain quantity of the unidentified minerals is implied, their concentration is not included in the concentrations of the identified minerals, which are summed to 100 percent.

\section{DRILLING MUD USAGE}

Drilling mud, containing montmorillonite and barite, was used on Leg 24 as follows:

\footnotetext{
${ }^{1}$ Institute of Geophysics and Planetary Physics, University of California Riverside, California, Contribution No. 73-78.
}

No mud was used at Sites 231, 232, 233, 235, or 236. Montmorillonite was used at Site 234 after pulling Core 14 and after pulling Core 15. Montmorillonite and barite were used at Site 237 after pulling Core 64 and before pulling Core 65 . Core 67 at Site 237 got stuck so montmorillonite was used. Montmorillonite and barite were used at Site 238 prior to cutting Cores 58 and 59. Montmorillonite alone was used after pulling Core 61, after Core 62, and after Core 63. Most samples submitted for diffraction analysis do not occur close to intervals in which drilling mud was used. In the case of samples from Site 234, Core 15, the montmorillonite content is normal and barite is absent. This suggests that contamination by drilling mud is unlikely.

\section{NOTE ON SUPPLEMENTARY SAMPLES}

Many samples originally submitted for X-ray diffraction analysis by Leg 24 scientists were so calcareous that insufficient insoluble residue remained in the $2-20 \mu$ and $<2 \mu$ size classes following decalcification. In order to obtain more mineral data, Leg 24 scientists submitted a suite of supplementary samples for analysis. Large, decalcified $2-20 \mu$ and $<2 \mu$ samples were processed, and the results from the supplementary samples appear in Tables 16 through 19. In almost all cases the supplementary samples were taken a few centimeters above or below an original sample. Hence, a supplementary sample in Tables 16 through 19 will show the same depth (in meters) below the sea floor as original samples in Tables 8 through 15 for which there was insufficient residue.

The original and supplementary data are presented separately because during supplementary sample preparation some $<2 \mu$ size material was consistently introduced into the $2-20 \mu$ samples by an inexperienced operator. This has resulted in inconsistencies between the original and supplementary data. The most obvious difference is a high montmorillonite content in the $2-20 \mu$ fraction. The data are reported here to provide qualitative mineralogic information on the insoluble residue of the supplementary samples.

\section{ACKNOWLEDGMENTS}

The writers wish to acknowledge the excellent work of Nicki D. Coursey in the interpretation of X-ray diffraction data, of Paul D. Johnson in X-ray data aquisition and data processing, and of Tom W. Halverson, Jr., in sample preparation. 
TABLE 1

Summary of X-Ray Mineralogy Samples, Sample Depths, Lithology, Age, and X-Ray Diffraction Results, Site 231

\begin{tabular}{|c|c|c|c|c|c|c|}
\hline Core, Section, & $\begin{array}{c}\text { Sample } \\
\text { Depth Below }\end{array}$ & & & $\begin{array}{c}\text { Bulk Sample } \\
\text { Major Constituent }\end{array}$ & $\begin{array}{c}2-20 \mu \text { Fraction } \\
\text { Major Constituent }\end{array}$ & $\begin{array}{c}<2 \mu \text { Fraction } \\
\text { Major Constituent }\end{array}$ \\
\hline Interval (cm) & Sea Floor (m) & Lithology & Age & $\begin{array}{lll}1 & 2 & 3\end{array}$ & $\begin{array}{lll}1 & 2 & 3\end{array}$ & $\begin{array}{lll}1 & 2 & 3\end{array}$ \\
\hline $2-2,68-70$ & 2.7 & $\begin{array}{l}\text { Unit I: } \\
\text { clay-bearing } \\
\text { nanno ooze }\end{array}$ & $\begin{array}{l}\text { Holocene to } \\
\text { Pleistocene }\end{array}$ & Calc. Quar. Plag. & Quar. Plag. Mica & Paly. Mica Mont. \\
\hline $4-2,46-48$ & 18.5 & $\begin{array}{l}\text { Unit II: } \\
\text { nanno ooze } \\
\text { with inter- } \\
\text { calated sand } \\
\text { horizons }\end{array}$ & $\begin{array}{l}\text { Pleistocene to } \\
\text { Pliocene }\end{array}$ & Calc. Arag. Quar. & Quar. Plag. Mica & Paly. Mica Kaol. \\
\hline $\begin{array}{l}31-4,69-71 \\
44-1,90-92 \\
61-6,127-129\end{array}$ & $\begin{array}{l}278.2 \\
397.4 \\
565.8\end{array}$ & $\begin{array}{l}\text { Unit V: } \\
\text { clay-bearing } \\
\text { nanno ooze }\end{array}$ & $\begin{array}{l}\text { Lower Miocene } \\
\text { through } \\
\text { Pliocene }\end{array}$ & $\begin{array}{l}\text { Calc. Paly. Quar. } \\
\text { Paly. Quar. Mont. } \\
\text { Calc. Paly. Quar. }\end{array}$ & $\begin{array}{ll}\text { Quar. Mica Plag. } \\
\text { Quar. Mica Plag. } \\
\text { Quar. Mica Paly. }\end{array}$ & $\begin{array}{l}\text { Paly. Mont. Kaol. } \\
\text { Paly. Mont. Mica } \\
\text { Paly. Mont. Mica }\end{array}$ \\
\hline
\end{tabular}

TABLE 2

Summary of X-Ray Mineralogy Samples, Sample Depths, Lithology, Age, and X-Ray Diffraction Results, Site 232

\begin{tabular}{|c|c|c|c|c|c|c|}
\hline Core, Section, & $\begin{array}{c}\text { Sample } \\
\text { Depth Below }\end{array}$ & & & $\begin{array}{c}\text { Bulk Sample } \\
\text { Major Constituent }\end{array}$ & $\begin{array}{c}2-20 \mu \text { Fraction } \\
\text { Major Constituent }\end{array}$ & $\begin{array}{c}<2 \mu \text { Fraction } \\
\text { Major Constituent }\end{array}$ \\
\hline Interval $(\mathrm{cm})$ & Sea Floor $(m)$ & Lithology & Age & $\begin{array}{lll}1 & 2 & 3\end{array}$ & $\begin{array}{lll}1 & 2 & 3\end{array}$ & $\begin{array}{lll}1 & 2 & 3\end{array}$ \\
\hline \multicolumn{7}{|l|}{ Hole 232} \\
\hline $\begin{array}{l}1-1,94-96 \\
13-1,74-76 \\
18-3,50-52\end{array}$ & $\begin{array}{r}0.9 \\
107.1 \\
158.0\end{array}$ & $\begin{array}{l}\text { Unit I: } \\
\text { nanno ooze } \\
\text { with occa- } \\
\text { sional quart- } \\
\text { zose sand } \\
\text { layers }\end{array}$ & $\begin{array}{l}\text { Late Miocene } \\
\text { through } \\
\text { Holocene }\end{array}$ & $\begin{array}{l}\text { Calc. Paly. } \\
\text { Quar. Plag. Calc. } \\
\text { Calc. Paly. Quar. }\end{array}$ & $\begin{array}{l}\text { Insufficient residue } \\
\text { Mica Quar. Plag. } \\
\text { Quar. Mica Plag. }\end{array}$ & $\begin{array}{l}\text { Paly. } \\
\text { Paly. Mont. Mica } \\
\text { Paly. Mont. Mica }\end{array}$ \\
\hline \multicolumn{7}{|l|}{ Hole 232A } \\
\hline $\begin{array}{l}1-4,32-34 \\
7-2,112-114 \\
9-5,126-128 \\
14-1,110-112\end{array}$ & $\begin{array}{l}163.8 \\
218.6 \\
242.3 \\
283.6\end{array}$ & $\begin{array}{l}\text { Unit II: } \\
\text { nanno ooze } \\
\text { with occa- } \\
\text { sional quart- } \\
\text { zose sand } \\
\text { layers }\end{array}$ & Late Miocene & $\begin{array}{l}\text { Calc. Quar. Paly. } \\
\text { Quar. Plag. Calc. } \\
\text { Calc. Paly. Mica } \\
\text { Quar. Plag. K-Fe }\end{array}$ & $\begin{array}{ll}\text { Quar. Plag. } & \text { K-Fe } \\
\text { Quar. Plag. } & \text { Mica } \\
\text { Quar. Mica } & \text { Plag. } \\
\text { Clin. } & \end{array}$ & $\begin{array}{l}\text { Mont. Kaol. Mica } \\
\text { Paly. Mont. Kaol. } \\
\text { Paly. Mont. Kaol. } \\
\text { Mont. Paly. Kaol. }\end{array}$ \\
\hline
\end{tabular}

TABLE 3

Summary of X-Ray Mineralogy Samples, Sample Depths, Lithology, Age, and X-Ray Diffraction Results, Site 233

\begin{tabular}{|c|c|c|c|c|c|c|}
\hline Core, Section, & $\begin{array}{c}\text { Sample } \\
\text { Depth Below }\end{array}$ & & & $\begin{array}{c}\text { Bulk Sample } \\
\text { Major Constituent }\end{array}$ & $\begin{array}{c}2-20 \mu \text { Fraction } \\
\text { Major Constituent }\end{array}$ & $\begin{array}{c}<2 \mu \text { Fraction } \\
\text { Major Constituent }\end{array}$ \\
\hline Interval $(\mathrm{cm})$ & Sea Floor $(m)$ & Lithology & Age & $\begin{array}{lll}1 & 2 & 3\end{array}$ & $\begin{array}{lll}1 & 2 & 3\end{array}$ & $\begin{array}{lll}1 & 2 & 3\end{array}$ \\
\hline $2-4,76-78$ & 10.3 & $\begin{array}{l}\text { Unit I: } \\
\text { clay-bearing } \\
\text { nanno ooze }\end{array}$ & $\begin{array}{l}\text { Pliocene through } \\
\text { Holocene }\end{array}$ & Calc. Paly. & Quar. Plag. Mica & Paly. Mica Kaol. \\
\hline $\begin{array}{l}8-4,128-130 \\
8-4,130-132 \\
11-4,91-93\end{array}$ & $\begin{array}{l}67.79 \\
67.81 \\
95.9\end{array}$ & $\begin{array}{l}\text { Unit II: micarb } \\
\text { and clay-bearing } \\
\text { nanno ooze }\end{array}$ & $\begin{array}{l}\text { Pliocene and } \\
\text { Pleistocene }\end{array}$ & $\begin{array}{l}\text { Calc. Paly. Quar. } \\
\text { Calc. Paly. Quar. } \\
\text { Calc. Paly. Quar. }\end{array}$ & $\begin{array}{ll}\text { Quar. Plag. Mica } \\
\text { Quar. Plag. Paly. } \\
\text { Quar. Plag. Paly. }\end{array}$ & $\begin{array}{l}\text { Paly. Mica Mont. } \\
\text { Paly. Mica } \\
\text { Paly. Mica }\end{array}$ \\
\hline $16-4,43-45$ & 142.9 & $\begin{array}{l}\text { Unit III: } \\
\text { micarb and } \\
\text { clay-bearing } \\
\text { nanno ooze }\end{array}$ & Pliocene & Calc. Paly. Quar. & Quar. Mica Plag. & Paly. Mica Mont. \\
\hline
\end{tabular}


TABLE 4

Summary of X-Ray Mineralogy Samples, Sample Depths, Lithology, Age, and X-Ray Diffraction Results, Site 234

\begin{tabular}{|c|c|c|c|c|c|c|}
\hline Core, Section, & $\begin{array}{c}\text { Sample } \\
\text { Depth Below }\end{array}$ & & & $\begin{array}{c}\text { Bulk Sample } \\
\text { Major Constituent }\end{array}$ & $\begin{array}{c}2-20 \mu \text { Fraction } \\
\text { Major Constituent }\end{array}$ & $\begin{array}{c}<2 \mu \text { Fraction } \\
\text { Major Constituent }\end{array}$ \\
\hline Interval $(\mathrm{cm})$ & Sea Floor $(m)$ & Lithology & Age & $\begin{array}{lll}1 & 2 & 3\end{array}$ & $\begin{array}{lll}1 & 2 & 3\end{array}$ & $\begin{array}{lll}1 & 2 & 3\end{array}$ \\
\hline $\begin{array}{l}1-2,89-91 \\
1-4,53-55\end{array}$ & $\begin{array}{l}2.4 \\
5.0\end{array}$ & $\begin{array}{l}\text { Unit I: } \\
\text { clay-bearing } \\
\text { nanno ooze }\end{array}$ & Plio-Pleistocene & $\begin{array}{l}\text { Calc. Paly. Quar. } \\
\text { Calc. Paly. Mont. }\end{array}$ & $\begin{array}{ll}\text { Quar. Plag. } & \text { K-Fe. } \\
\text { K-Fe. Plag. } & \text { Quar. }\end{array}$ & $\begin{array}{l}\text { Mont. Paly. Mica } \\
\text { Mont. Paly. Mica }\end{array}$ \\
\hline $6-6,140-142$ & 84.9 & $\begin{array}{l}\text { Unit III: } \\
\text { clay and } \\
\text { nanno ooze }\end{array}$ & Middle Miocene & Paly. Mont. Mica & K-Fe. Quar. Plag. & Mont. Paly. Kaol. \\
\hline $10-4,14-16$ & 166.1 & $\begin{array}{l}\text { Unit V: } \\
\text { clay and } \\
\text { nanno ooze }\end{array}$ & Lower Miocene & Calc. Mont. K-Fe. & K-Fe. Plag. Quar. & Mont. Kaol. \\
\hline $15-3,44-46$ & 240.9 & $\begin{array}{l}\text { Unit VI: } \\
\text { Clay ooze }\end{array}$ & Oligocene & Mont. Kaol. Paly. & K-Fe. Plag. Quar. & Mont. Kaol. Paly. \\
\hline
\end{tabular}

TABLE 5

Summary of X-Ray Mineralogy Samples, Sample Depths, Lithology, Age, and X-Ray Diffraction Results, Site 235

\begin{tabular}{|c|c|c|c|c|c|c|}
\hline Core, Section, & $\begin{array}{c}\text { Sample } \\
\text { Depth Below }\end{array}$ & & & $\begin{array}{c}\text { Bulk Sample } \\
\text { Major Constituent }\end{array}$ & $\begin{array}{c}2-20 \mu \text { Fraction } \\
\text { Major Constituent }\end{array}$ & $\begin{array}{c}<2 \mu \text { Fraction } \\
\text { Major Constituent }\end{array}$ \\
\hline Interval (cm) & Sea Floor $(m)$ & Lithology & Age & $\begin{array}{lll}1 & 2 & 3\end{array}$ & $\begin{array}{lll}1 & 2 & 3\end{array}$ & $\begin{array}{lll}1 & 2 & 3\end{array}$ \\
\hline $\begin{array}{l}1-2,94-96 \\
4-3,30-32\end{array}$ & $\begin{array}{r}2.4 \\
31.8\end{array}$ & $\begin{array}{l}\text { Unit I: } \\
\text { nanno ooze } \\
\text { and chalk }\end{array}$ & Pleistocene & $\begin{array}{l}\text { Calc. Paly. } \\
\text { Calc. Paly. }\end{array}$ & $\begin{array}{l}\text { Quar. K-Fe. Plag. } \\
\text { Quar. Plag. K-Fe. }\end{array}$ & $\begin{array}{l}\text { Mont. Paly. Mica } \\
\text { Mont. Paly. Mica }\end{array}$ \\
\hline $\begin{array}{l}5-3,60-62 \\
5-6,78-79 \\
5-6,89-90 \\
5-6,110-111 \\
10-2,108-110 \\
11-3,23-25 \\
11-3,38-40 \\
11-3,80-82\end{array}$ & $\begin{array}{r}70.1 \\
74.8 \\
74.9 \\
75.1 \\
221.1 \\
269.2 \\
269.4 \\
269.8\end{array}$ & $\begin{array}{l}\text { Unit II: } \\
\text { nanno chalk } \\
\text { mud to } \\
\text { slightly } \\
\text { calcareous } \\
\text { clay mud }\end{array}$ & $\begin{array}{l}\text { Upper Miocene } \\
\text { through } \\
\text { Pleistocene }\end{array}$ & $\begin{array}{l}\text { Arag. Calc. } \\
\text { Calc. } \\
\text { Mont. Paly. Quar. } \\
\text { Calc. Paly. } \\
\text { Calc. } \\
\text { Calc. Arag. } \\
\text { Mont. K-Fe. Paly. } \\
\text { Calc. }\end{array}$ & $\begin{array}{l}\text { Insufficient residue } \\
\text { Insufficient residue } \\
\text { Quar. Plag. K-Fe. } \\
\text { Insufficient residue } \\
\text { K-Fe. Quar. Plag. } \\
\text { Insufficient residue } \\
\text { K-Fe. Plag. Quar. } \\
\text { K-Fe. Plag. Quar. }\end{array}$ & $\begin{array}{ll}\text { Insufficient residue } \\
\text { Mont. Paly. Mica } \\
\text { Mont. Paly. Kaol. } \\
\text { Paly. Mica Kaol. } \\
\text { Mont. Paly. Kaol. } \\
\text { Mont. Paly. } \\
\text { Mont. Paly. Kaol. } \\
\text { Mont. K-Fe. Paly. }\end{array}$ \\
\hline
\end{tabular}


TABLE 6

Summary of X-Ray Mineralogy Samples, Sample Depths, Lithology, Age, and X-Ray Diffraction Results, Site 236

\begin{tabular}{|c|c|c|c|c|c|c|}
\hline Core, Section, & $\begin{array}{c}\text { Sample } \\
\text { Depth Below }\end{array}$ & & & $\begin{array}{c}\text { Bulk Sample } \\
\text { Major Constituent }\end{array}$ & $\begin{array}{c}2-20 \mu \text { Fraction } \\
\text { Major Constituent }\end{array}$ & $\begin{array}{c}<2 \mu \text { Fraction } \\
\text { Major Constituent }\end{array}$ \\
\hline Interval (cm) & Sea Floor $(m)$ & Lithology & Age & $\begin{array}{lll}1 & 2 & 3\end{array}$ & 3 & 3 \\
\hline $\begin{array}{l}3-2,68-70 \\
3-2,126-128 \\
5-3,41-43 \\
5-3,71-73 \\
5-6,118-120 \\
6-2,107-109 \\
8-3,88-90 \\
9-3,112-114 \\
12-6,40-42 \\
12-6,92-94 \\
15-4,94-96\end{array}$ & \begin{tabular}{r|}
18.2 \\
18.8 \\
38.4 \\
38.7 \\
43.7 \\
47.1 \\
67.4 \\
77.1 \\
109.4 \\
109.9 \\
135.4
\end{tabular} & $\begin{array}{l}\text { Unit II: } \\
\text { clay-bearing } \\
\text { nanno-ooze }\end{array}$ & $\begin{array}{l}\text { Lower Miocene } \\
\text { through } \\
\text { Pleistocene }\end{array}$ & $\begin{array}{l}\text { Calc. } \\
\text { Calc. } \\
\text { Calc. Arag. } \\
\text { Calc. Arag. } \\
\text { Calc. Arag. } \\
\text { Calc. } \\
\text { Calc. Mont. Quar. } \\
\text { Calc. Arag. } \\
\text { Calc. Arag. } \\
\text { Calc. } \\
\text { Calc. }\end{array}$ & $\begin{array}{l}\text { Insufficient residue } \\
\text { Quar. Plag. Mica } \\
\text { Insufficient residue } \\
\text { Insufficient residue } \\
\text { Insufficient residue } \\
\text { Quar. K-Fe. Plag. } \\
\text { Plag. Quar. K-Fe } \\
\text { Insufficient residue } \\
\text { Insufficient residue } \\
\text { Quar. Plag. K-Fe. } \\
\text { Quar. Plag. Mica }\end{array}$ & $\begin{array}{l}\text { Insufficient residue } \\
\text { Mont. Mica Paly. } \\
\text { Insufficient residue } \\
\text { Insufficient residue } \\
\text { Mont. Paly. Mica } \\
\text { Mont. Paly. Mica } \\
\text { Mont. Paly. Mica } \\
\text { Mont. Paly. } \\
\text { Mont. Paly. } \\
\text { Mont. Paly. Mica } \\
\text { Mont. Paly. Mica }\end{array}$ \\
\hline $\begin{array}{l}16-5,82-84 \\
19-2,88-90\end{array}$ & $\begin{array}{l}146.3 \\
170.4\end{array}$ & $\begin{array}{l}\text { Unit III: } \\
\text { nanno-bearing } \\
\text { clays }\end{array}$ & Miocene & $\begin{array}{l}\text { Mont. Paly. Quar. } \\
\text { Mont. Paly. K-Fe. }\end{array}$ & $\begin{array}{l}\text { Quar. Plag. K-Fe. } \\
\text { Phil. K-Fe. Quar. }\end{array}$ & $\begin{array}{l}\text { Mont. Paly. Quar. } \\
\text { Mont. Paly. Kaol. }\end{array}$ \\
\hline $\begin{array}{l}20-5,110-112 \\
22-4,89-90 \\
25-4,62-64\end{array}$ & $\begin{array}{l}184.6 \\
201.9 \\
230.1\end{array}$ & $\begin{array}{l}\text { Unit IV: } \\
\text { nanno ooze } \\
\text { with minor } \\
\text { forams, rads, } \\
\text { and clay }\end{array}$ & Oligocene & $\begin{array}{l}\text { Calc. } \\
\text { Calc. } \\
\text { Calc. }\end{array}$ & $\begin{array}{l}\text { Clin. Paly. } \\
\text { Insufficient residue } \\
\text { Insufficient residue }\end{array}$ & $\begin{array}{l}\text { Paly. Mont. } \\
\text { Mont. Gyps. } \\
\text { Insufficient residue }\end{array}$ \\
\hline $29-1,140-142$ & 264.4 & $\begin{array}{l}\text { Unit V: } \\
\text { clay-rich } \\
\text { nanno chalk }\end{array}$ & Eocene & Calc. & Clin. Quar. & Gyps. Mont. Paly. \\
\hline $33-1,98-100$ & 305.0 & $\begin{array}{l}\text { Unit VI: } \\
\text { clay-bearing } \\
\text { nanno chalk }\end{array}$ & Paleocene & Calc. & Clin. Mont. & Mont. Paly. \\
\hline
\end{tabular}

TABLE 7

Summary of X-Ray Mineralogy Samples, Sample Depths, Lithology, Age, and X-Ray Diffraction Results, Site 238

\begin{tabular}{|c|c|c|c|c|c|c|}
\hline Core, Section, & $\begin{array}{c}\text { Sample } \\
\text { Depth Below }\end{array}$ & & & $\begin{array}{c}\text { Bulk Sample } \\
\text { Major Constituent }\end{array}$ & $\begin{array}{c}2-20 \mu \text { Fraction } \\
\text { Major Constituent }\end{array}$ & $\begin{array}{c}<2 \mu \text { Fraction } \\
\text { Major Constituent }\end{array}$ \\
\hline Interval (cm) & Sea Floor (m) & Lithology & Age & $\begin{array}{lll}1 & 2 & 3\end{array}$ & $\begin{array}{lll}1 & 2 & 3\end{array}$ & 2 \\
\hline $\begin{array}{l}1-4,82-84 \\
5-4,103-105 \\
7-2,110-112 \\
7-2,119-121 \\
7-5,12-14 \\
14-2,76-78 \\
14-4,90-92 \\
15-5,76-77\end{array}$ & $\begin{array}{r}5.3 \\
43.5 \\
56.1 \\
56.2 \\
59.6 \\
122.3 \\
125.4 \\
136.4\end{array}$ & $\begin{array}{l}\text { Unit I: } \\
\text { nanno ooze } \\
\text { to foram-rich } \\
\text { nanno ooze } \\
\text { with minor } \\
\text { clay }\end{array}$ & $\begin{array}{l}\text { Upper Miocene } \\
\text { through } \\
\text { Quaternary }\end{array}$ & $\begin{array}{l}\text { Calc. } \\
\text { Calc. } \\
\text { Calc. } \\
\text { Calc. } \\
\text { Calc. } \\
\text { Calc. } \\
\text { Calc. } \\
\text { Calc. }\end{array}$ & $\begin{array}{l}\text { Insufficient residue } \\
\text { Insufficient residue } \\
\text { Insufficient residue } \\
\text { Insufficient residue } \\
\text { Insufficient residue } \\
\text { Insufficient residue } \\
\text { Insufficient residue } \\
\text { Insufficient residue }\end{array}$ & $\begin{array}{l}\text { Insufficient residue } \\
\text { Insufficient residue } \\
\text { Insufficient residue } \\
\text { Insufficient residue } \\
\text { Insufficient residue } \\
\text { Insufficient residue } \\
\text { Insufficient residue } \\
\text { Insufficient residue }\end{array}$ \\
\hline $\begin{array}{l}38-5,103-105 \\
39-5,97-99 \\
41-2,67-69 \\
49-2,100-102\end{array}$ & $\begin{array}{l}355.0 \\
364.5 \\
378.7 \\
455.0\end{array}$ & $\begin{array}{l}\text { Unit II: } \\
\text { nanno ooze } \\
\text { to foram-bear- } \\
\text { nanno ooze }\end{array}$ & $\begin{array}{l}\text { Upper Oligocene } \\
\text { through Middle } \\
\text { Miocene }\end{array}$ & $\begin{array}{l}\text { Calc. } \\
\text { Calc. } \\
\text { Calc. } \\
\text { Calc. }\end{array}$ & $\begin{array}{l}\text { Insufficient residue } \\
\text { Insufficient residue } \\
\text { Insufficient residue } \\
\text { Phil. Plag. K-Fe }\end{array}$ & $\begin{array}{l}\text { Gyps. Hali. Mont. } \\
\text { Insufficient residue } \\
\text { Insufficient residue } \\
\text { Mont. Phil. K-Fe. }\end{array}$ \\
\hline $\begin{array}{l}51-2,13-15 \\
51-4,70-72 \\
52-1,59-61 \\
52-3,2-3 \\
52-3,13-17 \\
53-2,6-8 \\
53-3,126-128 \\
53-4,67-69 \\
54-1,23-25 \\
54-1,84\end{array}$ & $\begin{array}{l}473.1 \\
476.7 \\
481.6 \\
484.0 \\
484.1 \\
492.1 \\
494-8 \\
495.7 \\
500.2 \\
500.8\end{array}$ & $\begin{array}{l}\text { Unit III: } \\
\text { nanno chalk } \\
\text { with inter- } \\
\text { calated } \\
\text { horizons of } \\
\text { volcanic ash } \\
\text { and zeolites }\end{array}$ & $\begin{array}{l}\text { Lower and } \\
\text { Upper Oligocene }\end{array}$ & $\begin{array}{l}\text { Calc. } \\
\text { Calc. } \\
\text { Calc. } \\
\text { Mont. K-Fe. Plag. } \\
\text { Calc. } \\
\text { Calc. } \\
\text { Calc. Phil. } \\
\text { Calc. } \\
\text { Calc. } \\
\text { Calc. Plag. Phil. }\end{array}$ & $\begin{array}{l}\text { Insufficient residue } \\
\text { K-Fe. Phil. Plag. } \\
\text { Phil. K-Fe. Plag. } \\
\text { K-Fe. Augi. Plag. } \\
\text { Mont. Plag. K-Fe. } \\
\text { Mont. Phil. Plag. } \\
\text { Phil. } \\
\text { Phil. Plag. Mont. } \\
\text { Insufficient residue } \\
\text { Insufficient residue }\end{array}$ & $\begin{array}{l}\text { Mont. Phil. K-Fe. } \\
\text { Mont. Phil. } \\
\text { Mont. Phil. K-Fe. } \\
\text { Mont. Augi. } \\
\text { Mont. } \\
\text { Mont. } \\
\text { Phil. Mont. } \\
\text { Mont. Phil. Plag. } \\
\text { Mont. Gyps. Phil. } \\
\text { Insufficient residue }\end{array}$ \\
\hline
\end{tabular}


TABLE 8

Results of X-Ray Diffraction Analysis from Site 231

\begin{tabular}{|c|c|c|c|c|c|c|c|c|c|c|c|c|c|c|c|c|c|c|}
\hline Core & $\begin{array}{c}\text { Cored } \\
\text { Interval } \\
\text { Below Sea } \\
\text { Floor (m) }\end{array}$ & $\begin{array}{l}\text { Sample Depth } \\
\text { Below Sea } \\
\text { Floor } \\
\text { (m) }\end{array}$ & 范 & 客 & ปु & ஹ் & 嫣 & 萠 & 蓠 & $\frac{a}{20}$ & 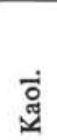 &  & 茓 & 菅 & त्ञ & 㤐 & 点 & $\frac{\pi}{\square}$ \\
\hline
\end{tabular}

Bulk Samples

$\begin{array}{rcrrrrrrrrrrrrrrrr}2 & 0.5-7.0 & 2.7 & 72.8 & 57.5 & 61.4 & 2.6 & - & 10.0 & 3.0 & 7.6 & 1.4 & 5.7 & 1.2 & - & 6.0 & - & 1.2 \\ 4 & 16.5-26.0 & 18.5 & 67.7 & 49.5 & 54.2 & 1.5 & 21.5 & 12.8 & - & 2.0 & - & 3.3 & - & - & 3.3 & 1.4 & - \\ 31 & 273.0-282.5 & 278.2 & 70.3 & 53.6 & 62.3 & 3.4 & - & 7.9 & 2.5 & 2.3 & 2.0 & 5.4 & 0.9 & 1.2 & 11.2 & 0.9 & - \\ 44 & 396.5-406.0 & 397.4 & 81.9 & 71.8 & 5.5 & 7.5 & - & 21.6 & 3.8 & 6.7 & 2.3 & - & 2.5 & 9.0 & 37.1 & 3.8 & - \\ 61 & 557.0-566.5 & 565.8 & 78.6 & 66.5 & 42.4 & - & - & 10.4 & 2.9 & 3.8 & - & 5.8 & 1.1 & 3.8 & 29.9 & - & -\end{array}$

2-20 $\mu$ Fractions

\begin{tabular}{|c|c|c|c|c|c|c|c|c|c|c|c|c|c|c|}
\hline 2 & $0.5-7.0$ & 2.7 & 75.5 & 61.7 & 3.4 & 36.4 & 10.9 & 24.4 & 1.4 & 16.1 & 3.5 & - & 1.1 & 2.9 \\
\hline 4 & $16.5-26.0$ & 18.5 & 97.9 & 96.7 & - & 43.0 & 8.4 & 22.7 & - & 13.7 & 2.2 & - & 6.6 & 3.4 \\
\hline 31 & $273.0-282.5$ & 278.2 & 69.1 & 51.7 & - & 35.9 & 12.7 & 17.8 & 2.1 & 20.4 & 3.8 & - & 7.3 & - \\
\hline 44 & $396.5-406.0$ & 397.4 & 73.9 & 59.2 & - & 28.3 & 10.3 & 14.1 & 4.0 & 22.3 & 2.4 & 12.6 & 6.0 & - \\
\hline 61 & $557.0-566.5$ & 565.8 & 74.0 & 59.3 & - & 35.7 & 10.6 & 13.9 & - & 20.8 & 3.2 & 14.8 & 1.1 & - \\
\hline
\end{tabular}

$<2 \mu$ Fractions

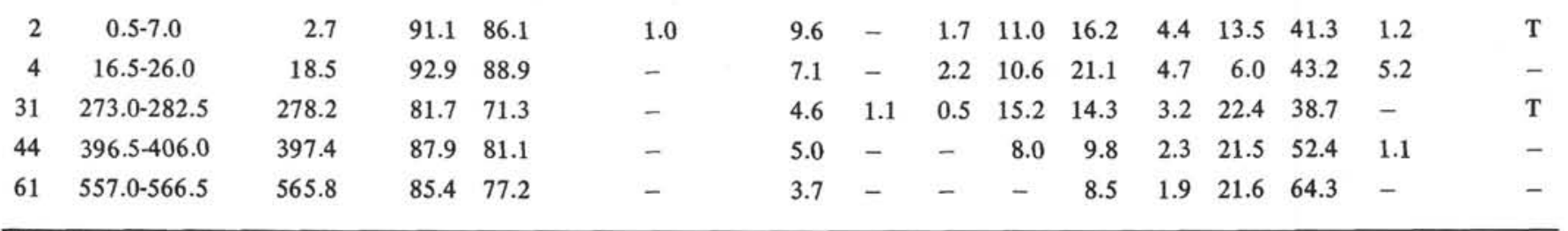

aU-1 Peaks at $5.74 \AA, 3.62 \AA$, and $8.02 \AA$. T $=$ trace.

TABLE 9

Results of X-Ray Diffraction Analysis from Hole 232

\begin{tabular}{|c|c|c|c|c|c|c|c|c|c|c|c|c|c|c|c|c|c|}
\hline Core & $\begin{array}{c}\text { Cored } \\
\text { Interval } \\
\text { Below Sea } \\
\text { Floor (m) }\end{array}$ & $\begin{array}{l}\text { Sample Depth } \\
\text { Below Sea } \\
\text { Floor } \\
\text { (m) }\end{array}$ & 巡 & 客 & गु & 응 & 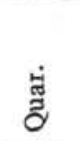 & 它 & 当 & $\begin{array}{l}\text { वें } \\
\text { ฯ }\end{array}$ & $\stackrel{\overbrace{}}{\Sigma}$ & 롱 & $\frac{\dot{\vec{J}}}{\stackrel{0}{\Sigma}}$ & 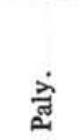 & 灾 & 容 & $\frac{\pi}{1}$ \\
\hline \multicolumn{18}{|c|}{ Bulk Samples } \\
\hline 1 & $0.0-2.5$ & 0.9 & 70.0 & 53.1 & 74.9 & 3.7 & 7.2 & - & 2.8 & - & 2.6 & - & - & 8.9 & - & - & - \\
\hline 13 & $107.0-116.5$ & 107.1 & 59.2 & 36.3 & 20.5 & 1.5 & 29.2 & 14.7 & 22.7 & - & 6.7 & 0.4 & - & - & - & 4.3 & - \\
\hline 18 & $154.5-164.0$ & 158.0 & 72.6 & 57.2 & 49.1 & 6.3 & 8.9 & 3.3 & 5.2 & 0.9 & 5.6 & 1.4 & 1.0 & 17.3 & 1.2 & - & $\mathrm{T}$ \\
\hline \multicolumn{18}{|c|}{$2-20 \mu$ Fractions } \\
\hline 13 & $107.0-116.5$ & 107.1 & 73.1 & 58.0 & & & 22.2 & 9.2 & 16.3 & & 24.7 & 4.5 & & 11.3 & 9.1 & 2.7 & \\
\hline 18 & $154.5-164.0$ & 158.0 & 68.5 & 50.8 & & & 32.0 & 8.9 & 18.5 & & 19.5 & 3.3 & & 12.8 & 5.1 & - & \\
\hline \multicolumn{18}{|c|}{$<2 \mu$ Fractions } \\
\hline 1 & $0.0-2.5$ & 0.9 & 90.9 & 85.8 & & & 3.7 & & & 4.9 & 5.4 & 1.9 & 5.8 & 78.2 & - & & $\mathrm{T}$ \\
\hline 13 & $107.0-116.5$ & 107.1 & 86.5 & 78.9 & & & 4.9 & & & 7.3 & 12.0 & 2.8 & 19.0 & 49.9 & 4.0 & & $\mathrm{~T}$ \\
\hline 18 & $154.5-164.0$ & 158.0 & 85.0 & 76.5 & & & 3.0 & & & 8.5 & 10.3 & 1.4 & 16.6 & 60.2 & - & & $\mathrm{T}$ \\
\hline
\end{tabular}

aU-1 Peaks at $5.74 \AA, 3.62 \AA$, and $8.02 \AA . T=$ trace. 
TABLE 10

Results of X-Ray Diffraction Analysis from Hole 232A

\begin{tabular}{|c|c|c|c|c|c|c|c|c|c|c|c|c|c|c|c|c|c|c|}
\hline Core & $\begin{array}{c}\text { Cored } \\
\text { Interval } \\
\text { Below Sea } \\
\text { Floor (m) }\end{array}$ & $\begin{array}{l}\text { Sample Depth } \\
\text { Below Sea } \\
\text { Floor } \\
\text { (m) }\end{array}$ & 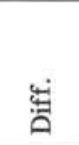 & 忘 & ల் & 융 & 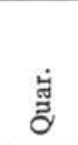 & $\frac{\dot{s}}{\dot{y}}$ & $\frac{a j}{\tilde{a}}$ & 迅 & 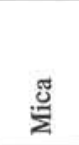 & 宅 & $\frac{\dot{\Xi}}{\dot{0}}$ & $\overrightarrow{\text { त्d }}$ & 苞 & 完 & 总 & $\stackrel{\pi}{7}$ \\
\hline 1 & $159.0-168.5$ & 163.8 & 80.3 & 69.2 & 49.2 & 3.9 & 13.2 & 4.1 & 8.0 & - & 7.6 & 0.7 & - & 12.4 & & 1.0 & - & $\mathrm{T}$ \\
\hline 7 & $216.0-225.5$ & 218.6 & 67.9 & 49.8 & 21.0 & 1.8 & 31.7 & 10.1 & 21.1 & - & 9.8 & 0.8 & - & - & & 1.2 & 2.5 & - \\
\hline 14 & $282.5-292.0$ & 283.6 & 66.1 & 47.0 & 9.9 & - & 37.8 & 13.5 & 19.5 & - & 8.9 & 0.4 & - & 7.3 & & 1.0 & 1.7 & - \\
\hline \multicolumn{19}{|c|}{ 2-20 $\mu$ Fractions } \\
\hline 1 & $159.0-168.5$ & 163.8 & 93.9 & 90.5 & & & 31.3 & 16.1 & 20.2 & - & 13.3 & 2.4 & & 8.5 & - & 6.1 & 2.1 & \\
\hline 7 & $216.0-225.5$ & 218.6 & 57.8 & 34.1 & & & 32.6 & 9.3 & 25.2 & - & 21.8 & 2.7 & & - & - & 5.9 & 2.6 & \\
\hline 1 & $159.0-168.5$ & 163.8 & 89.3 & 83.3 & & & 11.7 & & & 20.6 & 15.0 & 8.4 & 38.7 & - & & 5.7 & & - \\
\hline 7 & $216.0-225.5$ & 218.6 & 81.2 & 70.7 & & & 5.1 & & & 14.0 & 6.9 & 3.9 & 24.8 & 43.8 & & 1.5 & & - \\
\hline 9 & $235.0-244.5$ & 242.3 & 84.8 & 76.2 & & & 4.7 & & & 11.8 & 10.2 & 1.7 & 30.8 & 39.7 & & 1.1 & & $\mathrm{~T}$ \\
\hline 14 & $282.5-292.0$ & 283.6 & 83.0 & 73.5 & & & 3.8 & & & 15.1 & 6.6 & 2.5 & 43.0 & 29.0 & & - & & - \\
\hline
\end{tabular}

${ }^{\mathrm{a}} \mathrm{U}-1$ peaks at $5.74 \AA, 3.62 \AA$, and $8.02 \AA . \mathrm{T}=$ trace.

TABLE 11

Results of S-Ray Diffraction Analy sis from Site 233

\begin{tabular}{|c|c|c|c|c|c|c|c|c|c|c|c|c|c|c|c|c|c|}
\hline Core & $\begin{array}{c}\text { Cored } \\
\text { Interval } \\
\text { Below Sea } \\
\text { Floor (m) }\end{array}$ & $\begin{array}{l}\text { Sample Depth } \\
\text { Below Sea } \\
\text { Floor } \\
\text { (m) }\end{array}$ & 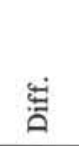 & 岑 & లુ & 응 & 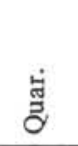 & 㭊 & $\frac{\pi}{2}$ & 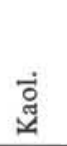 & ¿ّ & 总 & 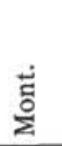 & 宊 & 完 & 亯 & $\frac{\pi}{1}$ \\
\hline \multicolumn{18}{|c|}{ Bulk Samples } \\
\hline 2 & $5.0-14.5$ & 10.30 & 63.0 & 42.2 & 74.2 & 4.0 & 5.9 & 1.5 & 2.3 & - & 3.1 & - & & 9.0 & - & & \\
\hline 8 & $62.0-71.5$ & $\begin{array}{l}67.79 \\
67.81\end{array}$ & $\begin{array}{l}71.0 \\
69.8\end{array}$ & $\begin{array}{l}54.7 \\
52.9\end{array}$ & $\begin{array}{l}48.5 \\
54.4\end{array}$ & $\begin{array}{l}5.6 \\
5.4\end{array}$ & $\begin{array}{r}11.2 \\
9.6\end{array}$ & $\begin{array}{l}4.8 \\
2.0\end{array}$ & $\begin{array}{l}7.1 \\
5.0\end{array}$ & - & $\begin{array}{l}5.0 \\
6.2\end{array}$ & $\begin{array}{l}1.0 \\
-\end{array}$ & & $\begin{array}{l}15.2 \\
17.5\end{array}$ & $\begin{array}{l}1.6 \\
-\end{array}$ & & \\
\hline 11 & $90.5-100.0$ & 95.90 & 64.1 & 43.9 & 50.5 & 4.7 & 11.6 & 1.6 & 5.9 & - & 5.7 & 1.1 & & 18.9 & - & & \\
\hline 16 & $138.0-147.5$ & 142.90 & 71.3 & 55.1 & 58.4 & 3.5 & 8.8 & 2.1 & 4.3 & 1.6 & 7.2 & - & & 12.5 & 1.4 & & \\
\hline \multicolumn{18}{|c|}{ 2-20 $\mu$ Fractions } \\
\hline 2 & $5.0-14.5$ & 10.30 & 71.3 & 55.2 & & 8.7 & 36.1 & 7.0 & 21.5 & & 12.0 & 2.8 & & 10.7 & - & 1.3 & \\
\hline 8 & $62.0-71.5$ & $\begin{array}{l}67.79 \\
67.81\end{array}$ & $\begin{array}{l}71.7 \\
73.7\end{array}$ & $\begin{array}{l}55.9 \\
59.0\end{array}$ & & - & $\begin{array}{l}31.9 \\
31.6\end{array}$ & $\begin{array}{l}8.0 \\
7.6\end{array}$ & $\begin{array}{l}21.7 \\
20.3\end{array}$ & & $\begin{array}{l}17.3 \\
15.5\end{array}$ & $\begin{array}{l}4.3 \\
3.0\end{array}$ & & $\begin{array}{l}11.0 \\
15.7\end{array}$ & $\begin{array}{l}4.8 \\
5.3\end{array}$ & $\begin{array}{l}1.1 \\
1.1\end{array}$ & \\
\hline 11 & $90.5-100.0$ & 95.90 & 73.5 & 58.6 & & - & 33.8 & 7.4 & 20.9 & & 13.8 & 2.8 & & 16.5 & 4.9 & - & \\
\hline 16 & $138.0-147.5$ & 142.90 & 73.5 & 58.5 & & - & 29.4 & 8.3 & 19.5 & & 22.4 & 3.8 & & 10.3 & 6.3 & - & \\
\hline \multicolumn{18}{|c|}{$<2 \mu$ Fractions } \\
\hline 2 & $5.0-14.5$ & 10.30 & 89.6 & 83.7 & & 1.2 & 4.8 & & & 8.0 & 12.9 & 2.9 & 6.9 & 63.4 & - & & $\mathrm{T}$ \\
\hline 8 & $62.0-71.5$ & $\begin{array}{l}67.79 \\
67.81\end{array}$ & $\begin{array}{l}86.6 \\
87.6\end{array}$ & $\begin{array}{l}79.1 \\
80.7\end{array}$ & & - & $\begin{array}{l}5.4 \\
3.5\end{array}$ & & & $\begin{array}{l}3.6 \\
3.9\end{array}$ & $\begin{array}{l}8.5 \\
8.5\end{array}$ & $\begin{array}{l}2.4 \\
2.9\end{array}$ & $\begin{array}{l}7.6 \\
6.2\end{array}$ & $\begin{array}{l}71.0 \\
74.2\end{array}$ & $\begin{array}{l}1.7 \\
0.9\end{array}$ & & - \\
\hline 11 & $90.5-100.0$ & 95.90 & 87.9 & 81.0 & & - & 3.7 & & & 6.2 & 10.0 & 1.8 & 7.1 & 70.2 & 1.1 & & - \\
\hline 16 & $138.0-147.5$ & 142.90 & 83.1 & 73.7 & & 1.1 & 5.1 & & & 6.1 & 14.4 & 1.9 & 7.6 & 61.4 & 2.4 & & - \\
\hline
\end{tabular}

${ }^{\mathrm{a}} \mathrm{U}-1$ peaks at $5.74 \AA, 3.62 \AA$, and $8.02 \AA . \mathrm{T}=$ trace. 
TABLE 12

Results of X-Ray Diffraction Analysis from Site 234

\begin{tabular}{|c|c|c|c|c|c|c|c|c|c|c|c|c|c|c|c|c|c|c|}
\hline Core & $\begin{array}{c}\text { Cored } \\
\text { Interval } \\
\text { Below Sea } \\
\text { Floor }(\mathrm{m})\end{array}$ & $\begin{array}{l}\text { Sample Depth } \\
\text { Below Sea } \\
\text { Floor } \\
\text { (m) }\end{array}$ & 焗 & 芒 & 远 & $\dot{\circ}$ & 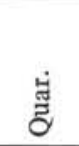 & 这 & घं & $\begin{array}{l}\overrightarrow{0} \\
\stackrel{3}{4}\end{array}$ & 旁 & 宅 & $\stackrel{\dot{\Xi}}{\dot{\Xi}}$ & 良 & 完 & 总 & $\frac{\pi}{1}$ & $\stackrel{\overbrace{}}{ָ}$ \\
\hline \multicolumn{19}{|c|}{ Bulk Samples } \\
\hline 1 & $0.0-9.5$ & $\begin{array}{l}2.4 \\
5.0\end{array}$ & $\begin{array}{l}78.6 \\
82.1\end{array}$ & $\begin{array}{l}66.6 \\
72.1\end{array}$ & $\begin{array}{l}60.0 \\
59.7\end{array}$ & $\begin{array}{l}2.4 \\
-\end{array}$ & $\begin{array}{l}9.4 \\
7.4\end{array}$ & $\begin{array}{l}2.0 \\
6.9\end{array}$ & $\begin{array}{l}3.6 \\
4.4\end{array}$ & $\begin{array}{l}2.3 \\
0.9\end{array}$ & $\begin{array}{l}5.9 \\
-\end{array}$ & & $\begin{array}{l}4.6 \\
8.1\end{array}$ & $\begin{array}{r}9.8 \\
12.6\end{array}$ & - & & $\frac{T}{-}$ & - \\
\hline 6 & $76.0-85.5$ & 84.9 & 89.2 & 83.1 & - & 2.1 & 12.1 & 6.1 & 3.2 & 9.5 & 12.9 & & 24.1 & 26.4 & 3.6 & & $\mathrm{~T}$ & - \\
\hline 10 & $161.5-171.0$ & 166.1 & 86.2 & 78.5 & 41.6 & - & 4.9 & 10.2 & - & 6.9 & - & & 29.7 & 6.7 & - & & $\mathrm{T}$ & $\mathrm{T}$ \\
\hline 15 & $237.5-247.0$ & 240.9 & 85.1 & 76.7 & - & - & 9.4 & 4.9 & 4.9 & 16.0 & 8.9 & & 43.5 & 12.5 & - & & - & - \\
\hline \multicolumn{19}{|c|}{ 2-20 $\mu$ Fractions } \\
\hline 1 & $0.0-9.5$ & $\begin{array}{l}2.4 \\
5.0\end{array}$ & $\begin{array}{l}79.2 \\
77.3\end{array}$ & $\begin{array}{l}67.5 \\
64.5\end{array}$ & & & $\begin{array}{l}41.4 \\
24.3\end{array}$ & $\begin{array}{l}17.6 \\
36.4\end{array}$ & $\begin{array}{l}25.2 \\
26.3\end{array}$ & - & $\begin{array}{r}12.5 \\
9.7\end{array}$ & $\begin{array}{l}2.3 \\
1.6\end{array}$ & & & $\overline{1.8}$ & $\begin{array}{l}1.0 \\
-\end{array}$ & & \\
\hline 6 & $76.0-85.5$ & 84.9 & 73.8 & 59.0 & & & 25.8 & 36.8 & 21.0 & 1.8 & 12.3 & 1.4 & & & 0.9 & - & & \\
\hline 10 & $161.5-171.0$ & 166.1 & 90.8 & 85.7 & & & 17.9 & 49.8 & 23.4 & 2.3 & 6.6 & - & & & - & - & & \\
\hline 15 & $237.5-247.0$ & 240.9 & 79.1 & 67.4 & & & 22.2 & 31.3 & 23.0 & 3.4 & 20.1 & - & & & - & - & & \\
\hline \multicolumn{19}{|c|}{$<2 \mu$ Fractions } \\
\hline 1 & $0.0-9.5$ & $\begin{array}{l}2.4 \\
5.0\end{array}$ & $\begin{array}{l}87.5 \\
91.6\end{array}$ & $\begin{array}{l}80.5 \\
86.9\end{array}$ & & $\begin{array}{l}1.7 \\
-\end{array}$ & $\begin{array}{l}6.6 \\
5.2\end{array}$ & $\overline{7.2}$ & & $\begin{array}{l}8.2 \\
7.4\end{array}$ & $\begin{array}{l}11.3 \\
19.1\end{array}$ & $\begin{array}{l}1.7 \\
-\end{array}$ & $\begin{array}{l}37.1 \\
30.3\end{array}$ & $\begin{array}{l}33.4 \\
29.3\end{array}$ & $\overline{1.4}$ & & $\overline{\mathrm{T}}$ & \\
\hline 6 & $76.0-85.5$ & 84.9 & 91.3 & 86.4 & & - & 6.9 & 8.0 & & 15.8 & 8.6 & 2.8 & 33.9 & 24.1 & - & & - & \\
\hline 10 & $161.5-171.0$ & 166.1 & 87.9 & 81.1 & & - & 2.2 & 3.9 & & 23.5 & - & - & 70.5 & - & - & & - & \\
\hline 15 & $237.5-247.0$ & 240.9 & 83.4 & 74.0 & & - & 3.4 & 1.0 & & 20.9 & 4.9 & - & 60.4 & 9.4 & - & & - & \\
\hline
\end{tabular}

${ }^{\mathrm{a}} \mathrm{U}-1$ peaks at $5.74 \AA, 3.62 \AA$, and $8.02 \AA . \mathrm{T}=$ trace.

$\mathrm{b}_{\mathrm{U}-2}$ peaks at $3.16 \AA, 5.57 \AA$, and $3.43 \AA$. 
TABLE 13

Results of X-Ray Diffraction Analysis from Site 235

\begin{tabular}{|c|c|c|c|c|c|c|c|c|c|c|c|c|c|c|c|c|c|c|c|}
\hline \multicolumn{20}{|c|}{ Bulk Samples } \\
\hline 1 & $0.0-9.5$ & 2.4 & 72.4 & 56.8 & 88.1 & - & - & 3.1 & - & - & 1.3 & - & & - & 7.5 & & - & $\mathrm{T}$ & - \\
\hline 4 & $28.5-38.0$ & 31.8 & 78.8 & 66.9 & 73.8 & 1.4 & - & 4.7 & 2.6 & 1.7 & 1.8 & 5.7 & & - & 8.4 & & - & $\mathrm{T}$ & - \\
\hline 10 & $218.5-228.0$ & 221.1 & 73.8 & 59.0 & 77.9 & - & - & 3.2 & 5.0 & 3.6 & 1.3 & - & & 4.9 & 4.1 & & - & - & - \\
\hline 11 & $266.0-275.5$ & $\begin{array}{l}269.2 \\
269.4 \\
269.8\end{array}$ & $\begin{array}{l}60.3 \\
89.3 \\
71.7\end{array}$ & $\begin{array}{l}37.9 \\
83.3 \\
55.7\end{array}$ & $\begin{array}{c}52.6 \\
- \\
82.2\end{array}$ & $\begin{array}{l}- \\
-\end{array}$ & $\begin{array}{c}47.4 \\
\overline{2} .6\end{array}$ & $\begin{array}{r}-\overline{12.5} \\
2.0\end{array}$ & $2 \overline{-} .5$ & $\begin{array}{l}\overline{8.7} \\
-\end{array}$ & $\begin{array}{l}\overline{5} .3 \\
-\end{array}$ & $\begin{array}{l}\overline{7.4} \\
2.2\end{array}$ & & $\begin{array}{r}\bar{r} .3 \\
5.1\end{array}$ & $\begin{array}{r}\overline{17.9} \\
5.9\end{array}$ & & $\begin{array}{l}\overline{1.4} \\
-\end{array}$ & $\begin{array}{l}\bar{T} \\
\bar{T}\end{array}$ & $\overline{\mathrm{T}}$ \\
\hline
\end{tabular}

2-20 $\mu$ Fractions

\begin{tabular}{|c|c|c|c|c|c|c|c|c|c|c|c|c|}
\hline 1 & $0.0-9.5$ & 2.4 & 86.1 & 78.2 & 28.6 & 21.7 & 19.4 & 3.7 & 6.2 & - & 17.4 & - \\
\hline 4 & $28.5-38.0$ & 31.8 & 86.6 & 79.1 & 32.9 & 19.0 & 20.7 & 5.2 & 14.1 & - & 7.1 & - \\
\hline 5 & $66.5-76.0$ & 74.9 & 83.1 & 73.6 & 32.4 & 17.6 & 26.2 & 3.5 & 8.9 & - & 6.6 & - \\
\hline 10 & $218.5-228.0$ & 221.1 & 79.2 & 67.6 & 20.4 & 34.0 & 19.5 & 3.3 & 10.2 & - & 6.6 & - \\
\hline 11 & $266.0-275.5$ & $\begin{array}{l}269.4 \\
269.8\end{array}$ & $\begin{array}{l}77.1 \\
92.9\end{array}$ & $\begin{array}{l}64.3 \\
88.9\end{array}$ & $\begin{array}{l}21.1 \\
22.7\end{array}$ & $\begin{array}{l}32.7 \\
28.2\end{array}$ & $\begin{array}{l}22.4 \\
23.6\end{array}$ & $\begin{array}{l}0.1 \\
-\end{array}$ & $\begin{array}{r}2.2 \\
19.6\end{array}$ & $\overline{1.5}$ & $\begin{array}{c}10.8 \\
-\end{array}$ & $\begin{array}{l}7.3 \\
-\end{array}$ \\
\hline
\end{tabular}

$<2 \mu$ Fractions

\begin{tabular}{|c|c|c|c|c|c|c|c|c|c|c|c|c|c|c|c|}
\hline 1 & $0.0-9.5$ & 2.4 & 87.9 & 81.1 & - & 5.1 & - & 11.4 & 13.7 & - & 41.4 & 27.5 & 1.0 & - & - \\
\hline 4 & $28.5-38.0$ & 31.8 & 77.9 & 65.5 & 1.9 & 5.6 & - & 12.0 & 13.0 & 2.0 & 35.3 & 30.2 & - & $\mathrm{T}$ & - \\
\hline 5 & $66.5-76.0$ & $\begin{array}{l}74.8 \\
74.9 \\
75.1\end{array}$ & $\begin{array}{l}87.2 \\
87.6 \\
93.0\end{array}$ & $\begin{array}{l}80.0 \\
80.6 \\
89.0\end{array}$ & - & $\begin{array}{l}3.6 \\
5.7 \\
7.8\end{array}$ & - & $\begin{array}{r}8.0 \\
10.2 \\
12.8\end{array}$ & $\begin{array}{r}12.9 \\
8.8 \\
23.1\end{array}$ & $\begin{array}{l}1.8 \\
\overline{3.1}\end{array}$ & $\begin{array}{c}50.5 \\
40.4 \\
-\end{array}$ & $\begin{array}{l}23.2 \\
34.9 \\
51.8\end{array}$ & $\begin{array}{l}- \\
\overline{1.4}\end{array}$ & $\begin{array}{l}- \\
-\end{array}$ & $\begin{array}{l}\mathrm{T} \\
\mathrm{T} \\
-\end{array}$ \\
\hline 10 & $218.5-228.0$ & 221.1 & 88.5 & 82.1 & - & 4.0 & 1.6 & 9.6 & 8.8 & - & 60.5 & 15.5 & - & - & $\mathrm{T}$ \\
\hline 11 & $266.0-275.5$ & $\begin{array}{l}269.2 \\
269.4 \\
269.8\end{array}$ & $\begin{array}{l}88.5 \\
86.3 \\
88.5\end{array}$ & $\begin{array}{l}82.0 \\
78.6 \\
82.0\end{array}$ & $\begin{array}{l}- \\
-\end{array}$ & $\begin{array}{l}3.4 \\
4.6 \\
3.8\end{array}$ & $\frac{-}{\overline{19} .1}$ & $\begin{array}{r}7.3 \\
12.4 \\
7.4\end{array}$ & $\begin{array}{l}6.5 \\
\overline{9.2}\end{array}$ & $\begin{array}{l}1.5 \\
\overline{1.7}\end{array}$ & $\begin{array}{l}52.2 \\
53.1 \\
41.1\end{array}$ & $\begin{array}{l}29.1 \\
28.7 \\
17.7\end{array}$ & $\overline{1.2}$ & $\begin{array}{l}\bar{T} \\
\mathrm{P}\end{array}$ & - \\
\hline
\end{tabular}

${ }^{a} \mathrm{U}-1$ peaks at $5.74 \AA, 3.62 \AA$, and $8.02 \AA . T=$ trace $; \mathrm{P}=$ present.

$\mathrm{b}_{\mathrm{U}}-2$ peaks at $3.16 \AA, 5.57 \AA$, and $3.43 \AA$. 
TABLE 14

Results of X-Ray Diffraction Analysis from Site 236

\begin{tabular}{|c|c|c|c|c|c|c|c|c|c|c|c|c|c|c|c|c|c|c|c|c|c|c|c|c|c|}
\hline Core & $\begin{array}{c}\text { Cored } \\
\text { Interval } \\
\text { Below Sea } \\
\text { Floor }(\mathrm{m})\end{array}$ & $\begin{array}{l}\text { Sample Depth } \\
\text { Below Sea } \\
\text { Floor } \\
\text { (m) }\end{array}$ &  & 客 & లే & 용 & 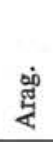 & ڤै & 官 & $\stackrel{a j}{a}$ & $\begin{array}{l}\overrightarrow{0} \\
\ddot{g}\end{array}$ & 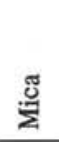 & 己े & है & ते & छ் & 覀 & 莽 & 棉 & 嵓 & 岕 & 离 & $\stackrel{\pi}{\square}$ & స̃ & $\begin{array}{l}0 \\
1 \\
0\end{array}$ \\
\hline
\end{tabular}

Bulk Samples

$3 \quad 16.0-25.5$

$5 \quad 35.0-44.5$

$6 \quad 44.5-54.0$

$8 \quad 63.5-73.0$

$9 \quad 73.0-82.5$

$12 \quad 101.5-111.0$

$15 \quad 130.0-139.5$

$16 \quad 139.5-149.0$

$19 \quad 168.0-177.5$

$20 \quad 177.5-187.0$

$22 \quad 196.5-206.0$

$25 \quad 225.0-234.5$

$29 \quad 263.0-272.5$

$33 \quad 301.0-306.5$

2-20 $\mu$ Fractions

$\begin{array}{rcrrr}3 & 16.0-25.5 & 18.8 & 98.3 & 97.3 \\ 6 & 44.5-54.0 & 47.1 & 85.7 & 77.6 \\ 8 & 63.5-73.0 & 67.4 & 90.5 & 85.2 \\ 12 & 101.5-111.0 & 109.9 & 88.2 & 81.5 \\ 15 & 130.0-139.5 & 135.4 & 90.4 & 85.0 \\ 16 & 139.5-149.0 & 146.3 & 82.9 & 73.3 \\ 19 & 168.0-177.5 & 170.4 & 82.1 & 72.0 \\ 20 & 177.5-187.0 & 184.6 & 72.0 & 56.3 \\ 29 & 263.0-272.5 & 264.4 & 66.9 & 48.3 \\ 33 & 301.0-306.5 & 305.0 & 61.1 & 39.1\end{array}$

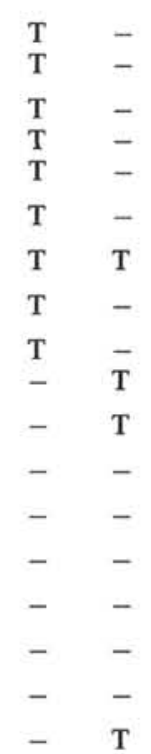

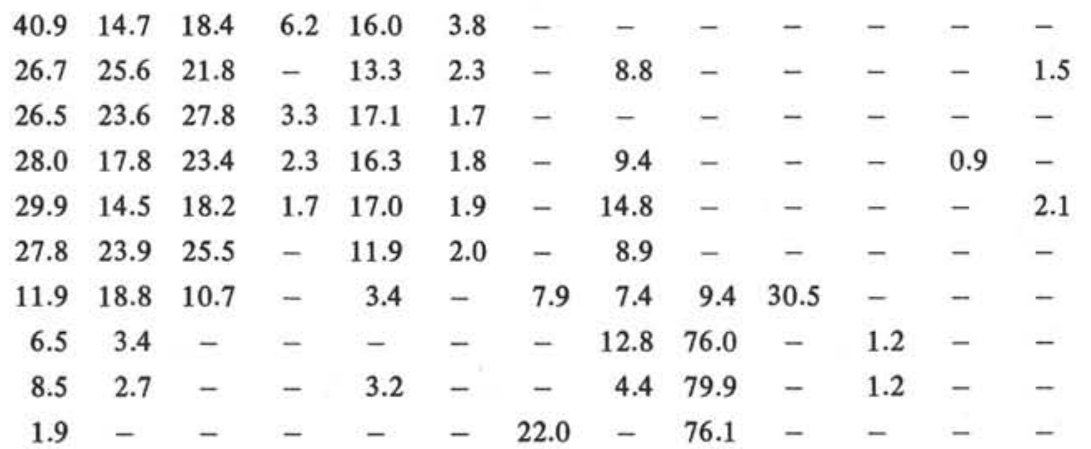


TABLE 14 - Continued

\begin{tabular}{|c|c|c|c|c|c|c|c|c|c|c|c|c|c|c|c|c|c|c|c|c|c|c|c|c|c|}
\hline Core & $\begin{array}{c}\text { Cored } \\
\text { Interval } \\
\text { Below Sea } \\
\text { Floor }(\mathrm{m})\end{array}$ & $\begin{array}{l}\text { Sample Depth } \\
\text { Below Sea } \\
\text { Floor } \\
\text { (m) }\end{array}$ & 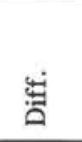 & 岁 & गु & $\dot{0}$ & 照 & 己ٌ & $\stackrel{\dot{0}}{\dot{\grave{1}}}$ & $\frac{0}{20}$ & $\begin{array}{l}\dot{0} \\
\check{g}\end{array}$ & हึ & 롱 & $\frac{\dot{E}}{\dot{E}}$ & خ্ & छ் & 立 & สี่ & 崖 & 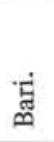 & 今. & ฮี่ & $\begin{array}{l}\pi \\
\square\end{array}$ & $\stackrel{\widetilde{T}}{\tilde{D}}$ & $\begin{array}{l}0 \\
i \\
p\end{array}$ \\
\hline \multicolumn{26}{|c|}{$<2 \mu$ Fractions } \\
\hline 3 & $16.0-25.5$ & 18.8 & 86.8 & 79.4 & & & & 8.0 & - & - & 11.6 & 21.9 & 2.0 & 37.4 & 19.1 & - & & & - & & - & - & - & - & - \\
\hline 5 & $35.0-44.5$ & 43.7 & 94.1 & 90.8 & & & & 3.5 & - & - & 5.7 & 11.0 & - & 65.4 & 14.5 & - & & & - & & - & - & - & - & - \\
\hline 6 & $44.5-54.0$ & 47.1 & 87.8 & 81.0 & & & & 4.3 & - & - & 9.2 & 14.3 & - & 51.6 & 20.6 & - & & & - & & - & - & $\mathrm{T}$ & - & - \\
\hline 8 & $63.5-73.0$ & 67.4 & 86.1 & 78.3 & & & & 4.3 & - & - & 7.2 & 12.5 & - & 50.6 & 25.5 & - & & & - & & - & - & $\mathrm{T}$ & - & - \\
\hline 9 & $73.0-82.5$ & 77.1 & 97.4 & 95.9 & & & & 4.5 & - & - & 7.5 & 6.6 & - & 62.7 & 14.7 & - & & & 4.0 & & - & - & - & - & - \\
\hline 12 & $101.5-111.0$ & $\begin{array}{l}109.4 \\
109.9\end{array}$ & $\begin{array}{l}93.9 \\
87.1\end{array}$ & $\begin{array}{l}90.5 \\
79.8\end{array}$ & & & & $\begin{array}{l}2.8 \\
4.7\end{array}$ & - & $\overline{-}$ & $\begin{array}{l}5.7 \\
4.5\end{array}$ & $\begin{array}{r}7.2 \\
11.4\end{array}$ & $\overline{1.9}$ & $\begin{array}{l}68.1 \\
52.8\end{array}$ & $\begin{array}{l}11.6 \\
24.7\end{array}$ & - & & & $\begin{array}{l}4.6 \\
-\end{array}$ & & - & $\overline{-}$ & $\overline{\mathrm{T}}$ & $\bar{T}$ & - \\
\hline 15 & $130.0-139.5$ & 135.4 & 86.6 & 79.1 & & & & 7.0 & - & - & 5.9 & 16.0 & 2.6 & 50.3 & 18.1 & - & & & - & & - & - & $\mathrm{T}$ & - & - \\
\hline 16 & $139.5-149.0$ & 146.3 & 90.3 & 84.8 & & & & 11.0 & 6.3 & 5.0 & 8.5 & 8.8 & - & 38.3 & 22.1 & - & & & - & & - & - & - & - & - \\
\hline 19 & $168.0-177.5$ & 170.4 & 88.3 & 81.7 & & & & 4.5 & 6.4 & - & 8.8 & 3.6 & - & 57.4 & 19.5 & - & & & - & & - & - & - & - & - \\
\hline 20 & $177.5-187.0$ & 184.6 & 90.5 & 85.1 & & & & 1.0 & - & - & - & - & - & 35.8 & 61.7 & 1.5 & & & - & & - & - & - & - & - \\
\hline 22 & $196.5-206.0$ & 201.9 & 91.7 & 87.0 & & & & 1.2 & - & - & - & - & - & 74.9 & - & - & & & - & & 20.7 & 3.2 & - & $\mathrm{P}$ & $\mathbf{P}$ \\
\hline 29 & $263.0-272.5$ & 264.4 & 89.1 & 82.9 & & & & 4.2 & - & - & - & - & - & 28.3 & 21.4 & - & & & - & & 35.5 & 10.5 & - & - & - \\
\hline 33 & $301.0-306.5$ & 305.0 & 79.9 & 68.6 & & & & 0.5 & - & - & - & - & - & 86.0 & 9.1 & 4.5 & & & - & & - & - & - & - & - \\
\hline
\end{tabular}

${ }^{\mathrm{a}} \mathrm{U}-1$ peaks at $5.74 \AA, 3.62 \AA$, and $8.02 \AA$. $\mathrm{T}=$ trace $\mathrm{P}=$ present.

$\mathrm{b}_{\mathrm{U}}-2$ peaks at $3.16 \AA, 5.57 \AA$, and $3.43 \AA$.

${ }^{c_{U}} \mathrm{U}-3$ peaks at $2.50 \AA, 2.67 \AA$, and $2.09 \AA$. 
TABLE 15

Results of X-Ray Diffraction Analysis from Site 238

\begin{tabular}{|c|c|c|c|c|c|c|c|c|c|c|c|c|c|c|c|c|c|c|c|c|}
\hline Core & $\begin{array}{c}\text { Cored } \\
\text { Interval } \\
\text { Below Sea } \\
\text { Floor }(\mathrm{m})\end{array}$ & $\begin{array}{l}\text { Sample Depth } \\
\text { Below Sea } \\
\text { Floor } \\
\text { (m) }\end{array}$ & 象 & 岁 & $\frac{\dot{j}}{\text { లु }}$ & हैं & 范 & $\frac{a}{a}$ & $\begin{array}{l}\dot{0} \\
\text { ¿ }\end{array}$ & 号 & 范 & 离 & 茝 & 蒁 & 岕 & 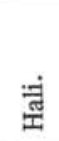 & 宠 & $\frac{\pi}{5}$ & $\stackrel{\overbrace{}}{~}$ & i \\
\hline \multicolumn{21}{|c|}{ Bulk Samples } \\
\hline 1 & $0.0-9.5$ & 5.3 & 55.3 & 30.2 & 100.0 & - & - & - & & & - & - & - & & & & - & - & $\mathrm{T}$ & - \\
\hline 5 & $38.0-44.0$ & 43.5 & 55.8 & 31.0 & 100.0 & - & - & - & & & - & - & - & & & & - & - & $\mathrm{T}$ & - \\
\hline 7 & $53.5-63.0$ & $\begin{array}{l}56.1 \\
56.2 \\
59.6\end{array}$ & $\begin{array}{l}54.2 \\
52.9 \\
56.4\end{array}$ & $\begin{array}{l}28.4 \\
26.4 \\
31.9\end{array}$ & $\begin{array}{l}100.0 \\
100.0 \\
100.0\end{array}$ & $\begin{array}{l}- \\
-\end{array}$ & $\begin{array}{l}- \\
-\end{array}$ & $\overline{-}$ & & & $\bar{z}$ & $\begin{array}{l}- \\
\overline{-}\end{array}$ & $\begin{array}{l}- \\
-\end{array}$ & & & & $\overline{-}$ & $\begin{array}{l}\bar{T} \\
T\end{array}$ & $\begin{array}{l}\mathrm{T} \\
\mathrm{T} \\
\mathrm{T}\end{array}$ & $\begin{array}{l}- \\
-\end{array}$ \\
\hline 14 & $120.0-129.5$ & $\begin{array}{l}122.3 \\
125.4\end{array}$ & $\begin{array}{l}54.4 \\
52.4\end{array}$ & $\begin{array}{l}28.8 \\
25.6\end{array}$ & $\begin{array}{l}100.0 \\
100.0\end{array}$ & $\overline{-}$ & - & - & & & - & - & - & & & & - & $\begin{array}{l}\mathrm{T} \\
\mathrm{T}\end{array}$ & $\begin{array}{l}\mathrm{T} \\
\mathrm{T}\end{array}$ & - \\
\hline 15 & $129.5-139.0$ & 136.3 & 49.8 & 21.5 & 100.0 & - & - & - & & & - & - & - & & & & - & $\mathrm{T}$ & $\mathrm{T}$ & - \\
\hline 38 & $348.0-357.5$ & 355.0 & 51.2 & 23.8 & 100.0 & - & - & - & & & - & - & - & & & & - & - & - & - \\
\hline 39 & $357.5-367.0$ & 364.5 & 51.6 & 24.3 & 98.7 & - & 1.3 & - & & & - & - & - & & & & - & $\mathrm{T}$ & - & - \\
\hline 41 & $376.5-386.0$ & 378.7 & 48.5 & 19.5 & 100.0 & - & - & - & & & - & - & - & & & & - & - & $\mathrm{T}$ & - \\
\hline 49 & $452.5-462.0$ & 455.0 & 49.5 & 21.1 & 99.5 & - & - & - & & & - & 0.5 & - & & & & - & - & $\mathrm{T}$ & - \\
\hline 51 & $471.5-481.0$ & $\begin{array}{l}473.1 \\
476.7\end{array}$ & $\begin{array}{l}51.2 \\
64.1\end{array}$ & $\begin{array}{l}25.1 \\
44.0\end{array}$ & $\begin{array}{r}100.0 \\
84.5\end{array}$ & - & $\overline{6} .6$ & $\overline{2} .9$ & & & $\overline{4} .2$ & - & $\overline{1.8}$ & & & & $\overline{-}$ & $\begin{array}{l}\mathrm{T} \\
-\end{array}$ & - & - \\
\hline 52 & $481.0-490.5$ & $\begin{array}{l}481.6 \\
484.0 \\
484.1\end{array}$ & $\begin{array}{l}51.8 \\
87.8 \\
59.3\end{array}$ & $\begin{array}{l}24.7 \\
80.9 \\
36.4\end{array}$ & $\begin{array}{l}98.3 \\
\overline{90.5}\end{array}$ & $\begin{array}{l}- \\
-\end{array}$ & $\begin{array}{r}\overline{15.2} \\
2.7\end{array}$ & $\begin{array}{r}\overline{12.1} \\
1.6\end{array}$ & & & $\begin{array}{r}\overline{63.6} \\
5.3\end{array}$ & $\begin{array}{l}- \\
\overline{-}\end{array}$ & $\begin{array}{l}1.7 \\
- \\
-\end{array}$ & & & & $\begin{array}{l}\overline{9} .1 \\
-\end{array}$ & $\begin{array}{l}- \\
-\end{array}$ & $\begin{array}{l}- \\
-\end{array}$ & $\begin{array}{l}- \\
\bar{T}\end{array}$ \\
\hline 53 & $490.5-500.0$ & $\begin{array}{l}492.1 \\
494.8 \\
495.7\end{array}$ & $\begin{array}{l}65.1 \\
68.1 \\
54.5\end{array}$ & $\begin{array}{l}45.4 \\
50.2 \\
28.9\end{array}$ & $\begin{array}{l}98.9 \\
65.1 \\
94.9\end{array}$ & $\begin{array}{l}- \\
\overline{-}\end{array}$ & $\overline{-}$ & $\begin{array}{l}- \\
\overline{-}\end{array}$ & & & $\begin{array}{l}1.1 \\
- \\
-\end{array}$ & $\begin{array}{l}- \\
-\end{array}$ & $\begin{array}{r}- \\
34.9 \\
5.1\end{array}$ & & & & $\overline{-}$ & $\begin{array}{l}\overline{\mathrm{T}} \\
-\end{array}$ & $\begin{array}{l}\text { T } \\
- \\
-\end{array}$ & $\begin{array}{l}- \\
-\end{array}$ \\
\hline 54 & $500.0-506.0$ & $\begin{array}{l}500.2 \\
500.8\end{array}$ & $\begin{array}{l}47.4 \\
84.6\end{array}$ & $\begin{array}{l}17.8 \\
75.9\end{array}$ & $\begin{array}{r}100.0 \\
40.3\end{array}$ & $\overline{1.5}$ & - & $\overline{28.0}$ & & & $\overline{11.1}$ & 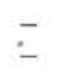 & $\overline{19.2}$ & & & & - & - & - & - \\
\hline \multicolumn{21}{|c|}{$<2-20 \mu$ Fractions } \\
\hline 49 & $452.5-462.0$ & 455.0 & 87.8 & 80.9 & & 1.2 & 15.9 & 22.1 & & - & - & 8.0 & 52.8 & & & & - & & & - \\
\hline 51 & $471.5-481.0$ & 476.7 & 82.5 & 72.7 & & 2.0 & 46.4 & 13.7 & & 1.6 & 8.3 & - & 28.0 & & & & - & & & - \\
\hline 52 & $481.0-490.5$ & $\begin{array}{l}481.6 \\
484.0 \\
484.1\end{array}$ & $\begin{array}{l}90.3 \\
93.4 \\
90.0\end{array}$ & $\begin{array}{l}84.9 \\
89.6 \\
84.4\end{array}$ & & $\begin{array}{l}0.7 \\
- \\
1.3\end{array}$ & $\begin{array}{l}23.2 \\
33.8 \\
19.6\end{array}$ & $\begin{array}{l}18.1 \\
25.4 \\
25.6\end{array}$ & & - & $\begin{array}{l}\overrightarrow{13.9} \\
35.6\end{array}$ & $\begin{array}{l}1.7 \\
- \\
-\end{array}$ & $\begin{array}{c}56.3 \\
\overline{17} .9\end{array}$ & & & & 26.9 & & & $\begin{array}{l}- \\
\bar{P}\end{array}$ \\
\hline 53 & $490.5-500.0$ & $\begin{array}{l}492.1 \\
494.8 \\
495.7\end{array}$ & $\begin{array}{l}89.0 \\
70.6 \\
77.4\end{array}$ & $\begin{array}{l}82.8 \\
54.1 \\
64.7\end{array}$ & & $\begin{array}{l}\overline{1} \\
\overline{1} .1\end{array}$ & $\begin{array}{l}- \\
-\end{array}$ & $\begin{array}{c}13.1 \\
\overline{23.3}\end{array}$ & & $\begin{array}{l}- \\
-\end{array}$ & $\begin{array}{c}70.9 \\
\overline{21.1}\end{array}$ & $\begin{array}{l}\overline{7} \\
\overline{7} .0\end{array}$ & $\begin{array}{r}15.9 \\
100.0 \\
47.5\end{array}$ & & & & $\begin{array}{l}- \\
\overline{-}\end{array}$ & & & - \\
\hline
\end{tabular}


TABLE 15 - Continued

\begin{tabular}{|c|c|c|c|c|c|c|c|c|c|c|c|c|c|c|c|c|c|c|c|c|}
\hline Core & $\begin{array}{c}\text { Cored } \\
\text { Interval } \\
\text { Below Sea } \\
\text { Floor (m) }\end{array}$ & $\begin{array}{l}\text { Sample Depth } \\
\text { Below Sea } \\
\text { Floor } \\
(\mathrm{m})\end{array}$ & 茗 & 客 & తુ & ప્ّ & 完 & $\stackrel{\pi}{2}$ & 宛 & 氙 & 莌 & छี่ & 咅 & สี่ & 令 & 竎 & 宥 & $\frac{\pi}{1}$ & $\stackrel{\overbrace{}}{\tilde{T}}$ & $\stackrel{0}{\square}$ \\
\hline \multicolumn{21}{|c|}{$<2 \mu$ Fractions } \\
\hline 38 & $348.0-357.5$ & 355.0 & 93.8 & 90.4 & & 3.7 & - & - & - & - & 22.1 & - & - & - & 39.9 & 34.3 & - & & & - \\
\hline 49 & $452.5-462.0$ & 455.0 & 86.9 & 79.5 & & 1.7 & 8.5 & 4.7 & - & 2.2 & 64.6 & 1.6 & 16.8 & - & - & - & - & & & - \\
\hline 51 & $471.5-481.0$ & $\begin{array}{l}473.1 \\
476.7\end{array}$ & $\begin{array}{l}97.8 \\
71.3\end{array}$ & $\begin{array}{l}96.6 \\
55.1\end{array}$ & & $\begin{array}{l}4.4 \\
-\end{array}$ & $\begin{array}{c}14.6 \\
-\end{array}$ & $\begin{array}{l}7.2 \\
-\end{array}$ & - & - & $\begin{array}{l}46.9 \\
83.7\end{array}$ & - & $\begin{array}{l}26.9 \\
15.2\end{array}$ & $\overline{1.1}$ & - & - & - & & & - \\
\hline 52 & $481.0-490.5$ & $\begin{array}{l}481.6 \\
484.0 \\
484.1\end{array}$ & $\begin{array}{l}90.2 \\
83.8 \\
88.1\end{array}$ & $\begin{array}{l}84.7 \\
74.7 \\
81.4\end{array}$ & & $\begin{array}{l}0.8 \\
- \\
-\end{array}$ & $\begin{array}{r}13.9 \\
3.3 \\
7.2\end{array}$ & $\begin{array}{l}- \\
4.3 \\
-\end{array}$ & $\begin{array}{l}- \\
-\end{array}$ & $\begin{array}{l}- \\
-\end{array}$ & $\begin{array}{l}71.0 \\
83.9 \\
92.8\end{array}$ & $\begin{array}{l}- \\
-\end{array}$ & $\begin{array}{l}14.3 \\
- \\
-\end{array}$ & $\begin{array}{l}- \\
-\end{array}$ & $\begin{array}{l}- \\
-\end{array}$ & $\begin{array}{l}- \\
- \\
-\end{array}$ & $\begin{array}{l}- \\
8.5 \\
-\end{array}$ & & & $\overline{-}$ \\
\hline 53 & $490.5-500.0$ & $\begin{array}{l}492.1 \\
494.8 \\
495.7\end{array}$ & $\begin{array}{l}84.6 \\
97.4 \\
87.8\end{array}$ & $\begin{array}{l}75.9 \\
95.9 \\
81.0\end{array}$ & & $\begin{array}{l}- \\
-\end{array}$ & - & $\begin{array}{l}- \\
\overline{7} .5\end{array}$ & $\begin{array}{l}- \\
\overline{-}\end{array}$ & $\begin{array}{l}- \\
-\end{array}$ & $\begin{array}{l}95.3 \\
41.5 \\
74.0\end{array}$ & $\begin{array}{l}- \\
-\end{array}$ & $\begin{array}{r}4.7 \\
58.5 \\
10.1\end{array}$ & $\begin{array}{l}- \\
-\end{array}$ & $\begin{array}{l}- \\
\overline{6} .9\end{array}$ & $\begin{array}{l}- \\
\overline{1.4}\end{array}$ & $\begin{array}{l}- \\
-\end{array}$ & & & - \\
\hline 54 & $500.0-506.0$ & 500.2 & 90.1 & 84.6 & & 2.1 & - & - & - & - & 62.4 & - & 13.4 & - & 14.0 & 8.1 & - & & & - \\
\hline
\end{tabular}

${ }^{\mathrm{a}} \mathrm{U}-1$ peaks at $5.74 \AA, 3.62 \AA$, and $8.02 \AA . T=$ trace.

$\mathrm{b}_{\mathrm{U}}-2$ peaks at $3.16 \AA, 5.57 \AA$, and $3.43 \AA$.

${ }^{c} \mathrm{U}-4$ broad peak at $3.56 \AA$. $\mathrm{P}=$ present. 
TABLE 16

Results of X-Ray Diffraction Analysis of Supplemental Samples from Site 232

\begin{tabular}{|c|c|c|c|c|c|c|c|c|c|c|c|c|c|}
\hline Core & Depth & $\begin{array}{l}\text { Sample Depth } \\
\text { Below } \\
\text { Sea Floor } \\
\text { (m) }\end{array}$ & Diffuse & $\begin{array}{l}\text { Amorphous } \\
\text { Scattering }\end{array}$ & $\dot{\circ}$ & 嵌 & 这 & 递 & $\stackrel{\text { ֻீ }}{\Sigma}$ & 袉 & 竞 & $\stackrel{\text { 岂 }}{\text { ¿ }}$ & 츌 \\
\hline \multicolumn{14}{|c|}{ 2-20 $\mu$ Fractions } \\
\hline 1 & $0-2.5$ & 0.9 & 65.9 & 46.7 & 12.2 & 37.6 & 6.8 & 21.0 & 16.6 & 4.7 & 1.2 & & \\
\hline \multicolumn{14}{|c|}{$<2 \mu$ Fractions } \\
\hline 1 & $0-2.5$ & 0.9 & 89.9 & 84.2 & & 11.7 & & & 23.8 & 3.4 & & 8.6 & 52.6 \\
\hline
\end{tabular}

TABLE 17

Results of X-Ray Diffraction Analysis of Supplemental Samples from Site 235

\begin{tabular}{|c|c|c|c|c|c|c|c|c|c|c|c|c|c|c|c|}
\hline Core & - Depth & $\begin{array}{l}\text { Sample Depth } \\
\text { Below } \\
\text { Sea Floor } \\
\text { (m) }\end{array}$ & Diffuse & $\begin{array}{l}\text { Amorphous } \\
\text { Scattering }\end{array}$ & 융 & हैं & 荘 & $\frac{\frac{\pi}{\pi}}{\pi}$ & $\stackrel{\stackrel{3}{2}}{\Sigma}$ & 혈 & ڤ્ & 官 & 家 & 迥 & $\stackrel{\overrightarrow{0}}{\Sigma}$ \\
\hline \multicolumn{16}{|c|}{ 2-20 $\mu$ Fractions } \\
\hline 5 & $66.5-76.6$ & 70.1 & 68.5 & 50.8 & 2.1 & 37.4 & 5.8 & 21.0 & 23.7 & 6.9 & & 3.1 & & & \\
\hline 5 & $66.5-76.0$ & 74.8 & 70.6 & 54.0 & 16.8 & 39.1 & & 18.6 & 17.5 & 5.4 & & 2.7 & & & \\
\hline 5 & $66.5-76.0$ & 75.1 & 72.5 & 57.0 & 6.7 & 36.4 & 5.0 & 22.3 & 17.9 & 5.4 & & 4.3 & 2.0 & & \\
\hline 11 & $266.0-275.5$ & 269.2 & 74.0 & 59.4 & 14.6 & 25.4 & 2.0 & 13.7 & 16.5 & 4.8 & 20.3 & 2.7 & & & \\
\hline \multicolumn{16}{|c|}{$<2 \mu$ Fractions } \\
\hline 5 & $66.5-76.0$ & 70.1 & 86.6 & 79.1 & & 19.5 & & 4.5 & 19.9 & 4.9 & 38.4 & 1.0 & & & 11.8 \\
\hline 5 & $66.5-76.0$ & 74.8 & 89.4 & 83.5 & 2.4 & 17.7 & & 3.9 & 22.6 & 4.9 & 41.2 & & & & 7.2 \\
\hline 5 & $66.5-76.0$ & 75.1 & 90.4 & 85.0 & & 15.0 & & 2.8 & 16.0 & 4.2 & 44.3 & 3.0 & & & 14.8 \\
\hline 11 & $266.0-275.5$ & 269.2 & 89.7 & 83.8 & 0.9 & 12.7 & & 2.8 & 16.8 & 3.0 & 53.2 & 1.3 & & 1.5 & 7.8 \\
\hline
\end{tabular}


TABLE 18

Results of X-Ray Diffraction Analysis of Supplemental Samples from Site 236

\begin{tabular}{|c|c|c|c|c|c|c|c|c|c|c|c|c|c|c|c|c|c|c|}
\hline Core & Depth & $\begin{array}{l}\text { Sample Depth } \\
\text { Below } \\
\text { Sea Floor } \\
\text { (m) }\end{array}$ & Diffuse & $\begin{array}{l}\text { Amorphous } \\
\text { Scattering }\end{array}$ & $\dot{\circ}$ & हैं & 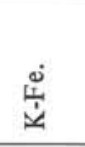 & $\frac{00}{2}$ & 离 & $\frac{\pi}{2}$ & है &  & 㝴 & 离 & 岕 & 房 & त्त & 퐆 \\
\hline \multicolumn{19}{|c|}{ 2-20 $\mu$ Fractions } \\
\hline 3 & $16.0-25.5$ & 18.2 & - & - & & 21.4 & 12.8 & 20.2 & 7.6 & 17.8 & & 17.8 & 2.3 & & & & & \\
\hline 3 & $16.0-25.5$ & 18.8 & 77.6 & 65.1 & & 33.4 & 13.1 & 18.7 & 2.2 & 22.9 & 4.3 & & & & & 5.3 & & \\
\hline 5 & $35.0-44.5$ & 38.4 & - & - & & 12.7 & 18.2 & 19.1 & 4.8 & 14.1 & 2.8 & 24.0 & 4.4 & & & & & \\
\hline 5 & $35.0-44.5$ & 38.7 & 74.6 & 60.3 & 15.2 & 23.8 & 19.4 & 21.8 & 2.6 & 13.9 & 2.2 & & 1.1 & & & & & \\
\hline 5 & $35.0-44.5$ & 43.7 & - & - & 1.2 & 19.8 & 22.0 & 31.6 & 5.8 & 8.2 & & & 11.5 & & & & & \\
\hline 9 & $73.0-82.5$ & 77.1 & 82.8 & 73.1 & 55.7 & 8.2 & 9.1 & 9.6 & & 3.9 & & & 1.9 & 7.5 & 4.1 & & & \\
\hline 12 & $101.5-111.0$ & 109.4 & 89.6 & 83.8 & 34.6 & 16.1 & 13.8 & 12.3 & & 5.5 & & & 2.4 & 7.7 & 7.7 & & & \\
\hline 22 & $196.5-206.0$ & 201.9 & 99.0 & 98.5 & & 20.3 & & 44.3 & & & & & & & & 35.3 & & \\
\hline 25 & $225.0-234.5$ & 230.1 & 96.0 & 93.8 & & 6.4 & & 16.3 & & 13.9 & & 14.8 & & & & 48.6 & & \\
\hline \multicolumn{19}{|c|}{$<2 \mu$ Fractions } \\
\hline 3 & $16.0-25.5$ & 18.2 & 89.9 & 84.1 & 3.2 & 9.4 & & & 8.0 & 10.7 & & 50.9 & & & & & 17.8 & \\
\hline 3 & $16.0-25.5$ & 18.8 & 88.4 & 81.8 & & 14.7 & 6.3 & 6.4 & 6.9 & 15.8 & 2.2 & 26.4 & & & & 3.1 & 18.2 & \\
\hline 5 & $35.0-44.5$ & 38.4 & 90.6 & 85.3 & 10.4 & 5.5 & & 4.5 & 7.8 & 13.0 & & 40.0 & & & & & 18.7 & \\
\hline 5 & $35.0-44.5$ & 38.7 & 90.3 & 84.9 & 2.4 & 13.4 & 11.6 & 8.1 & 4.0 & 11.3 & 2.8 & 33.5 & & & & & 13.0 & \\
\hline 5 & $35.0-44.5$ & 43.7 & 92.6 & 88.5 & 7.9 & 6.0 & & & 7.0 & 11.7 & & 38.1 & & & & & 20.3 & 9.1 \\
\hline 9 & $73.0-82.5$ & 77.1 & 93.3 & 89.5 & 5.2 & 5.4 & & 3.5 & 6.7 & 8.6 & & 40.2 & & & 8.5 & & 18.4 & 3.4 \\
\hline 12 & $101.5-111.0$ & 109.4 & 89.7 & 83.9 & & 5.5 & & & 6.5 & 8.7 & & 50.1 & & & 5.2 & & 14.0 & 10.1 \\
\hline 22 & $196.5-206.0$ & 201.9 & 88.8 & 82.5 & & & & & & & & 98.1 & & & & 1.9 & & \\
\hline 25 & $225.0-234.5$ & 230.1 & 86.9 & 79.6 & & 1.4 & & & 2.8 & 5.7 & & 78.1 & & & 2.7 & 3.3 & & 5.1 \\
\hline
\end{tabular}


TABLE 19

Results of X-Ray Diffraction Analysis of Supplemental Samples from Site 238

\begin{tabular}{|c|c|c|c|c|c|c|c|c|c|c|c|c|c|c|c|c|c|c|}
\hline Core & Depth & $\begin{array}{l}\text { Sample Depth } \\
\text { Below } \\
\text { Sea Floor } \\
\text { (m) }\end{array}$ & Diffuse & $\begin{array}{l}\text { Amorphous } \\
\text { Scattering }\end{array}$ & $\dot{\circ}$ & 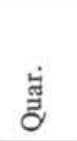 & 謧 & 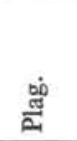 & 灾 & 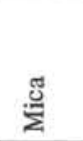 & हैं & हี่ & 竎 & 蒒 &  & ले & $\sum_{0}^{\infty}$ & 苂 \\
\hline \multicolumn{19}{|c|}{$2-20 \mu$ Fractions } \\
\hline 1 & $0-9.5$ & 5.3 & 93.6 & 89.9 & & 23.1 & & 17.5 & 6.3 & 15.0 & 3.1 & & & & 35.0 & & & \\
\hline 5 & $38.0-44.0$ & 43.5 & 94.0 & 90.6 & & 30.6 & & 8.2 & 7.2 & 15.4 & & & & 10.9 & 27.8 & & & \\
\hline 7 & $53.5-63.0$ & 56.1 & 92.4 & 88.1 & & 24.9 & & & 4.7 & 16.2 & & & & 21.5 & 32.7 & & & \\
\hline 7 & $53.5-63.0$ & 56.2 & - & - & & 14.1 & & & 4.2 & 8.0 & & 35.2 & 1.7 & 22.7 & 14.1 & & & \\
\hline 7 & $53.5-63.0$ & 59.6 & 96.3 & 94.2 & & 18.9 & & 12.8 & 9.7 & 13.7 & & & & & 44.8 & & & \\
\hline 14 & $120.0-129.5$ & 125.4 & - & - & 6.9 & 7.5 & & & & 51.9 & & & & 29.4 & 4.3 & & & \\
\hline 38 & $348.0-357.5$ & 355.0 & 94.9 & 92.1 & & 11.5 & 15.5 & 25.2 & 4.3 & 12.6 & & 22.6 & & & 8.3 & & & \\
\hline 39 & $357.5-367.0$ & 364.5 & - & - & & 2.4 & & 11.1 & & 3.9 & & 13.4 & 17.7 & 51.5 & & & & \\
\hline 41 & $376.5-386.0$ & 378.7 & - & - & & 4.2 & & & & & & & 36.0 & 59.8 & & & & \\
\hline 51 & $471.5-481.0$ & 473.1 & - & - & & 2.7 & & & & & & & 5.3 & 92.0 & & & & \\
\hline 54 & $500.0-506.0$ & 500.2 & - & - & & 2.0 & & 26.6 & & & & 14.2 & 11.0 & 46.1 & & & & \\
\hline \multicolumn{19}{|c|}{$<2 \mu$ Fractions } \\
\hline 1 & $0-9.5$ & 5.3 & 96.5 & 94.5 & & 7.9 & & & 5.9 & 13.7 & & 32.4 & 2.8 & & 10.1 & 27.1 & & \\
\hline 5 & $38.0-44.0$ & 43.5 & 96.3 & 94.2 & 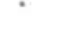 & 9.4 & & & 11.3 & 11.1 & & 55.7 & & & 12.5 & & & \\
\hline 7 & $53.5-63.0$ & 56.1 & 97.2 & 95.6 & & 7.5 & & & 8.3 & 14.1 & & 36.3 & & 8.0 & 9.7 & 16.2 & & \\
\hline 7 & $53.5-63.0$ & 56.2 & 90.8 & 85.7 & 3.5 & 2.5 & & & 4.0 & 10.0 & & 71.3 & & & 6.1 & & & 2.6 \\
\hline 7 & $53.5-63.0$ & 59.6 & 97.5 & 96.0 & & 8.9 & & & 16.4 & 32.8 & & 29.1 & & & 12.8 & & & \\
\hline 14 & $120.0-129.5$ & 122.3 & 97.1 & 95.4 & & 6.1 & & 7.6 & 14.0 & & & 65.1 & & & 7.2 & & & \\
\hline 14 & $120.0-129.5$ & 125.4 & 95.3 & 92.6 & & 5.2 & & & 12.5 & & & 73.1 & & & 9.2 & & & \\
\hline 15 & $129.5-139.0$ & 136.3 & 98.5 & 97.7 & & & & & & & & 100.0 & & & & & & \\
\hline 38 & $348.0-357.5$ & 355.0 & 89.6 & 83.7 & & 3.2 & & & 2.2 & & & 86.9 & & & & & 1.9 & 5.7 \\
\hline 39 & $357.5-367.0$ & 364.5 & 88.2 & 81.6 & & 1.4 & 6.3 & & & 16.2 & & 69.5 & & 6.6 & & & & \\
\hline 41 & $376.5-386$ & 378.7 & 90.1 & 84.5 & & 2.2 & 1.9 & & & 13.1 & & 78.4 & & 4.4 & & & & \\
\hline 51 & $471.5-481.0$ & 473.1 & - & - & & 3.5 & & 7.7 & & & & 59.0 & 3.1 & 26.7 & & & & \\
\hline 54 & $500.0-506.0$ & $\begin{array}{l}500.2 \\
500.8\end{array}$ & $8 \overline{7} .1$ & $\overline{79.8}$ & & $\begin{array}{l}4.5 \\
0.7\end{array}$ & 8.6 & $\begin{array}{r}2.8 \\
11.6\end{array}$ & & & & $\begin{array}{l}77.3 \\
43.5\end{array}$ & 1.6 & $\begin{array}{l}13.7 \\
22.2\end{array}$ & & & & \\
\hline
\end{tabular}

